\title{
Numerical Study on MHD Free Convection and Mass Transfer Flow Past a Vertical Flat Plate.
}

\author{
S. F. Ahmmed ${ }^{1}$, S. Mondal ${ }^{2}$ and A. Ray ${ }^{3}$ \\ 1,2,3 Mathematics Discipline, Khulna University, Bangladesh
}

\begin{abstract}
Two dimensional free convection and mass transfer flow of an incompressible, viscous and electrically conducting fluid past a continuously moving vertical flat plate in the presence of heat source, thermal diffusion, large suction and under the influence of uniform magnetic field applied normal to the flow is studied numerically. In this research work we make the governing equations dimensionless by appropriate coordinate transformation. We also consider the effect of heat source parameter and soret number for the above mentioned problem. Then these dimensionless momentum, energy and concentration equations are solved numerically by using explicit finite difference technique with the help of a computer programming language Compaq Visual Fortran 6.6. The obtained results of this study have been discussed for the different values of the well know parameters with different time step. The results are discussed in detailed with the help of graphs and tables to observe the effect of different parameters.
\end{abstract}

Key words- MHD, Thermal diffusion, Heat source parameter and Free convection.

\section{INTRODUCTION}

Magneto-hydrodynamic (MHD) is the branch of continuum mechanics which deals with the flow of electrically conducting fluids in electric and magnetic fields. Many natural phenomena and engineering problems are worth being subjected to an MHD analysis. Furthermore, Magneto-hydrodynamic (MHD) has attracted the attention of a large number of scholars due to its diverse applications. In engineering it finds its application in MHD pumps, MHD bearings etc.Free convection flows are of great interest in a number of industrial applications such as fiber and granular insulation, geothermal systems etc. Convection in porous media has applications in geothermal energy recovery, oil extraction, thermal energy storage and flow through filtering devices. The phenomena of mass transfer are also very common in theory of stellar structure and observable effects are detectable, at least on the solar surface. The study of effects of magnetic field on free convection flow is important in liquid-metal, electrolytes and ionized gases. The thermal physics of hydromagnetic problems with mass transfer is of interest in power engineering and metallurgy. The study of flows through porous media became of great interest due to its wide application in many scientific and engineering problems. Such type of flows can be observed in the movement of underground water resources, for filtration and water purification processes, the motion of natural gases and oil through oil reservoirs in petroleum engineering and so on. The velocity is usually so small and the flow passages are so narrow that laminar flow may be assumed without hesitation. Rigorous analysis of the flow is not possible because the shape of the individual flow passages is so varied and so complex. Recently researchers like R.K singh and A.k Singh [1] Chaudhary and Arpita [2] studied about MHD free convection heat and mass transfer in a vertical plate or sometimes oscillating plate. An analysis is performed to study the effect of thermal diffusing fluid past an infinite vertical porous plate with Ohmic dissipation by Reddy and Rao [3]. The combined effect of viscous dissipation, Joule heating, transpiration, heat source, thermal diffusion and Hall current on the hydro-Magnetic free convection and mass transfer flow of an electrically conducting, viscous, homogeneous, incompressible fluid past an infinite vertical porous plate are discussed by Singh et. al. [4]. An extensive contribution on heat and mass transfer flow has been made by Gebhart [5] to highlight the insight on the phenomena. Gebhart and Pera [6] studied heat and mass transfer flow under various flow situations. Therefore several authors, viz. Raptis and Soundalgekar [7], Jha and Singh [8] have paid attention to the study of MHD free convection and mass transfer flows. Abdusattar [9] also analyzed about MHD free convection through an infinite vertical plate. Acharya et. al.[10] have presented an analysis to study MHD effects on free convection and mass transfer flow through a porous medium with constant auction and constant heat flux considering Eckert number as a small perturbation parameter. This is the extension of the work of Bejan and Khair [11] under the influence of magnetic field. A numerical solution of unsteady free convection and mass transfer flow is presented by Alam and Rahman [12] when a viscous, incompressible fluid flows along an infinite vertical porous plate embedded in a porous medium is considered. N. P. Shingh [13] study about almost the similar problem to this work. 
In our present work the MHD free convection effect of large suction on heat and mass transfer flow past a vertical flat plate has been investigated by numerically which are obtained by employing the explicit finite difference technique with the help of a computer programming language Compaq Visual Fortran 6.6.

\section{GOVERNING EQUATIONS}

$$
\begin{aligned}
& \frac{\partial u}{\partial x}+\frac{\partial v}{\partial y}=0 \\
& \frac{\partial u}{\partial t}+u \frac{\partial u}{\partial x}+v \frac{\partial v}{\partial y}=\vartheta \frac{\partial^{2} u}{\partial y^{2}}+g \beta\left(T-T_{\infty}\right)+g \beta^{*}\left(C-C_{\infty}\right)-\frac{\sigma^{\prime} B_{0}^{2}(x)}{\rho} u \\
& \frac{\partial T}{\partial t}+u \frac{\partial T}{\partial x}+v \frac{\partial T}{\partial y}=\frac{K}{\rho C_{p}} \frac{\partial^{2} T}{\partial y^{2}}+Q\left(T-T_{\infty}\right) \\
& \frac{\partial C}{\partial t}+u \frac{\partial C}{\partial x}+v \frac{\partial C}{\partial y}=D_{M} \frac{\partial^{2} C}{\partial y^{2}}+D_{T} \frac{\partial^{2} T}{\partial y^{2}}
\end{aligned}
$$

The boundary conditions relevant to the problem are

$$
\begin{array}{ll}
u=U_{0}, \quad v=v_{0}(x), \quad T=T_{w}, \quad C=C_{w} & \text { at } \quad y=0 \\
u=0, \quad v=0, \quad T=T_{\infty}, \quad C=C_{\infty} \quad \text { as } \quad y \rightarrow \infty
\end{array}
$$

Where $u$ and $v$ are velocity components along $x$-axis and $y$-axis respectively, $g$ is acceleration due to gravity, $T$ is the temperature. $T_{w}$ is the wall temperature, $T_{\infty}$ isthe temperature of the uniform flow, $K$ is thermal conductivity, $\sigma^{\prime}$ is the electrical conductivity, $D_{M}$ is the molecular diffusivity, $U_{0}$ is the uniform velocity, $C$ is the concentration of species, $C_{\infty}$ is the concentration of species for uniform flow, $B_{0}(x)$ is the uniform magnetic field, $C_{P}$ is the specific at constant pressure, $Q$ is the constant heat source(absorption type), $D_{T}$ is the thermal diffusivity, $C_{w}$ is the mean concentration, $C(x)$ is variable concentration at the plate, $v_{0}(x)$ is the suction velocity, $\rho$ is the density, $\vartheta$ is the kinematic viscosity, $\beta$ is the volumetric coefficient of thermal expansion and $\beta^{*}$ is the volumetric coefficient of thermal expansion with concentration and the other symbols have their usual meaning.

\section{MATHEMATICAL FORMULATION}

Since the solutions of the governing equations (1)-(4) under the initial conditions (5) will be based on the finite difference method it is required to make the said equations dimensionless. For this purpose we now introduce the following dimensionless quantities

$$
X=x \frac{U_{0}}{v}, Y=y \frac{U_{0}}{v}, U=\frac{u}{U_{0}}, V=\frac{v}{U_{0}}, \tau=t \frac{U_{0}^{2}}{v}, T=T_{\infty}+\left(T_{w}-T_{\infty}\right) \theta, C=C_{\infty}+\left(C_{w}-C_{\infty}\right) \phi
$$

For the above dimensionless variable we have

$u=U U_{0}, v=V U_{0}$

Now using these relations we have substituted the values of the derivatives in to the equations (1)- (4) and after simplifications we obtain the following Non Linear coupled partial differentiate equation in terms of dimensionless variables, 
The continuity equation

$$
\frac{\partial U}{\partial X}+\frac{\partial V}{\partial Y}=0
$$

The momentum equation

$$
\frac{\partial U}{\partial t}+U \frac{\partial U}{\partial X}+V \frac{\partial V}{\partial Y}=\frac{\partial^{2} U}{\partial Y^{2}}+G r \theta+G m \phi-M U
$$

The Energy equation

$$
\frac{\partial \theta}{\partial t}+U \frac{\partial \theta}{\partial X}+V \frac{\partial \theta}{\partial Y}=\frac{1}{\operatorname{Pr}} \frac{\partial^{2} \theta}{\partial Y^{2}}+S \theta
$$

The Concentration equation

$$
\frac{\partial \phi}{\partial t}+U \frac{\partial \phi}{\partial X}+V \frac{\partial \phi}{\partial Y}=\frac{1}{S c} \frac{\partial^{2} \phi}{\partial Y^{2}}+\frac{D_{T}}{v} S_{0} \frac{\partial^{2} \theta}{\partial Y^{2}}
$$

Boundary conditions

$$
\begin{aligned}
& U=1, \quad V=\frac{V_{0}}{v_{0}}, \theta=1 \phi=1 \text { at } Y=0 \\
& U=0, \quad V=0, \quad \theta=0, \phi=0 \text { at } Y \rightarrow \infty
\end{aligned}
$$

\section{NUMERICAL SOLUTIONS}

In this section, we attempt to solve the governing second order nonlinear coupled dimensionless partial differential equations with the associated initial and boundary conditions. For solving a transient free convection flow with mass transfer past a semi-infinite plate, Callahan and Marner(1976) used the explicit finite difference method which is conditionally stable. On the contrary, the same problem was studied by Soundalgekar and Ganesan(1980) by an implicit finite difference method which is unconditionally stable. The only difference method between the two methods is that the implicit method being unconditionally stable is less expansive from the point of view of computer time. However, these two methods respectively employed by Callahan and Marner(1976) and Soundalgekar and Ganesan(1980) produced the same results. From the concept of the above discussion, for simplicity the explicit finite difference method has been used to solve equations.

Here we consider that the plate of height $X_{\max }=100$ i.e. $X$ varies from 0 to 100 and regard $Y_{\max }=25$ as corresponding to $Y \rightarrow \infty$ i.e. $Y$ varies from 0 to 25. There are $m=100$ and $n=100$ grid spacing in the $X$ and $Y$ directions respectively.

It is assumed that $\Delta X, \Delta Y$ are constant mesh sizes along $X$ and $Y$ directions respectively and taken as follows, $\Delta X=1.00(0 \leq x \leq 100$

$$
\Delta Y=0.2(0 \leq y \leq 25
$$

With the smaller time-step, $\Delta \tau=0.005$

Now using the finite difference method we convert our governing equations in the following form

Continuity equation

$$
\frac{U_{i, j}-U_{i-1, j}}{\Delta X}+\frac{V_{i, j}-V_{i, j-1}}{\Delta Y}=0
$$

Momentum equation

$$
\frac{U_{i, j}^{\prime}-U_{i, j}}{\Delta \tau}+U_{i, j} \frac{U_{i, j}-U_{i-1, j}}{\Delta X}+V_{i, j} \frac{V_{i, j}-V_{i, j-1}}{\Delta Y}=\frac{U_{i, j+1}-2 U_{i, j}+U_{i, j-1}}{(\Delta Y)^{2}}+G r \theta_{i, j}+G m \phi_{i, j}-M U_{i, j}
$$

Energy equation

$$
\frac{\theta_{i, j}^{\prime}-\theta_{i, j}}{\Delta \tau}+U_{i, j} \frac{\theta_{i, j}-\theta_{i-1, j}}{\Delta X}+V_{i, j} \frac{\theta_{i, j+1}-\theta_{i, j}}{\Delta Y}=\frac{1}{\operatorname{Pr}} \frac{\theta_{i, j+1}-2 \theta_{i, j}+\theta_{i, j-1}}{(\Delta Y)^{2}}+S \theta_{i, j}
$$

Concentration equation

$$
\frac{\phi_{i, j}^{\prime}-\phi_{i, j}}{\Delta \tau}+U_{i, j} \frac{\phi_{i, j}-\phi_{i-1, j}}{\Delta X}+V_{i, j} \frac{\phi_{i, j+1}-\phi_{i, j}}{\Delta Y}=\frac{1}{S c} \frac{\phi_{i, j+1}-2 \phi_{i, j}+\phi_{i, j-1}}{(\Delta Y)^{2}}+\frac{D_{T}}{v} S_{0} \frac{\theta_{i, j+1}-2 \theta_{i, j}+\theta_{i, j-1}}{(\Delta Y)^{2}}
$$


And the initial and boundary conditions with the finite difference scheme are

$U_{i, j}^{0}=0, V_{i, j}^{0}=\frac{V_{0}}{v_{0}}, \theta^{0}{ }_{i, j}=1, \phi_{i, j}^{0}=1 \quad$ at $y=0$

$U^{n}{ }_{i, j}=0, V^{n}{ }_{i, j}=0, \theta_{i, j}^{n}=0, \phi_{i, j}^{n}=0 \quad$ at $y \rightarrow \infty$

Here the subscripts i and designate the grid points with $x$ and $y$ coordinates respectively.

\section{RESULTS AND DISCUSSIONS}

To observe the physical situation of the problem under study, the velocity field, temperature field, concentration field are discussed by assigning numerical values to the parameters encountered into the corresponding equations. To be realistic, the values of magnetic parameter $(M=1.0,3.0,5.0)$, the values of Prandtl number $(\operatorname{Pr})$ are chosen for air $(\operatorname{Pr}=0.71)$, ammonia $(\operatorname{Pr}=0.90)$, water $(\operatorname{Pr}=7.0)$. the values of Schmidt number $(S c)$ are chosen for hydrogen $(S c=0.22)$, water-vapor $(S c=0.60)$, carbondioxeid $(S c=0.96)$ at $25^{\circ} \mathrm{C}$ and one atmosphere pressure. Grashof number for heat transfer is chosen to be $G r=1.0,3.0,5.0$ and modified Grashof number for mass transfer $G m=1.0,3.0,5.0$. Heat source parameter $(S=0.01,0.05,0.08)$ and Soret number $\left(S_{o}=0.1,0.5,1.0\right)$ are chosen arbitrarily.

The velocity profiles for different values of the above parameters are illustrated in Figure-1 to Figure- 8 . The temperature profiles for different values of the parameters are described in Figure- 9 and Figure-10. Also the concentration profiles for different values of the above parameters are expressed in Figure-11 to Figure-12. All the velocity, temperature and concentration profiles are given against the co-ordinate variable $(Y)$.

The Figure-1 depicts the velocity distributions for different values of magnetic parameter $M$. In this figure we observe that the velocity decreases with the increases of magnetic parameter.

The Figure- 2 demonstrates the velocity distributions for different values of Prandtl number Pr. From this figure we have noticed that the velocity decreases with the increases of Prandtl number. Physically this is true because the increase in the Prandtl number due to increasing the viscosity of the fluid which makes the fluid tick and hence a decrease in the velocity of fluid.

The Figure-3 represents the velocity distributions for different values of Schmidt number Sc. By this figure it is noticed that the velocity decreases with the increases of Schmidt number.

The velocity distributions for different values of Grashof number $G r$ are shown in the Figure-4. This figure is marked that the velocity increases with the increases of Grashof number.

The Figure-5 described the velocity distributions for different values of modified Grashof number $\mathrm{Gm}$. From this figure it is observed that the velocity increases with the increases of modified Grashof number $\mathrm{Gm}$.

The Figure-6 expressed the velocity distributions for different values of heat source parameter $S$. From this figure it is obtained that the velocity increases with the increases of heat source parameter $S$.

The velocity distributions for different values of Soret number $S_{o}$ are described in Figure-7. It is observed that the velocity increases with the increases of Soret number $S_{o}$.

The velocity distribution for different time parameter $\tau$ is explained in Figure-8. It is observed that the velocity increases with the increases of time.

We see from the Figure-9 that the temperature decreases with the increases of different Prandtl number Pr.

From the Figure-10 it is shown that the temperature increases with the increases of different heat source parameter $S$.

From the Figure-11 it is observed that the concentration profiles are decreases with the increases of different Schmidt number $S c$.

We observed from the Figure-12 that the concentration increases with the increases of different Soret number $S_{o}$ 


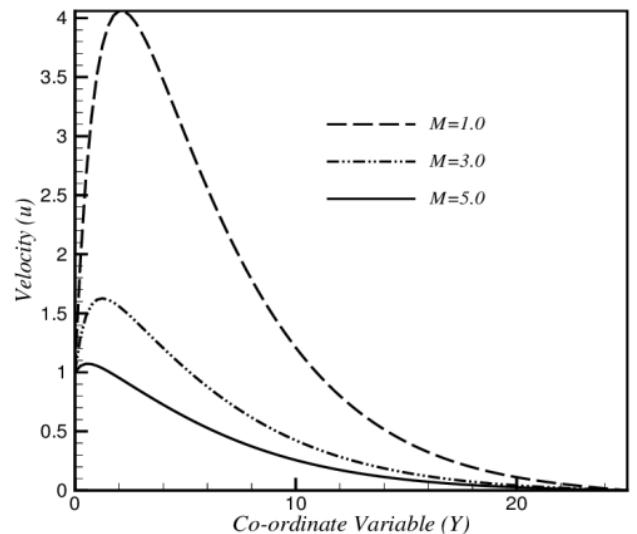

Fig. 1- Variation of velocity $u$ for different values of magnetic parameter $M$ for $\tau=10$ when $G r=3.0$, $G m=3.0, S=0.01, S_{o}=1.0, S c=0.22$ and $P r=0.71$

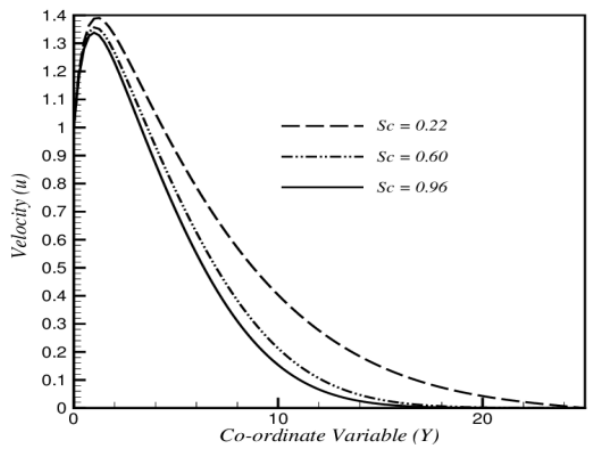

Fig. 3- Variation of velocity $u$ for different values of Schmidt number $S c$ at $\tau=10$ when $G r=2.0$, $G m=3.0, M=3.0, S_{o}=1.0, S=0.01$ and $P r=0.71$

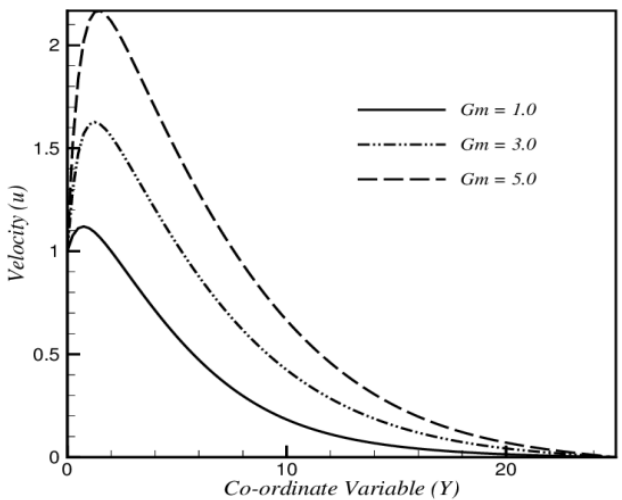

Fig. 5- Variation of velocity $u$ for different values of Modified Grashof number $\mathrm{Gm}$ at $\tau=10$ when $G r=3.0, M=3.0, S_{o}=1.0, S=0.01$, $P r=0.71$ and $S c=0.22$

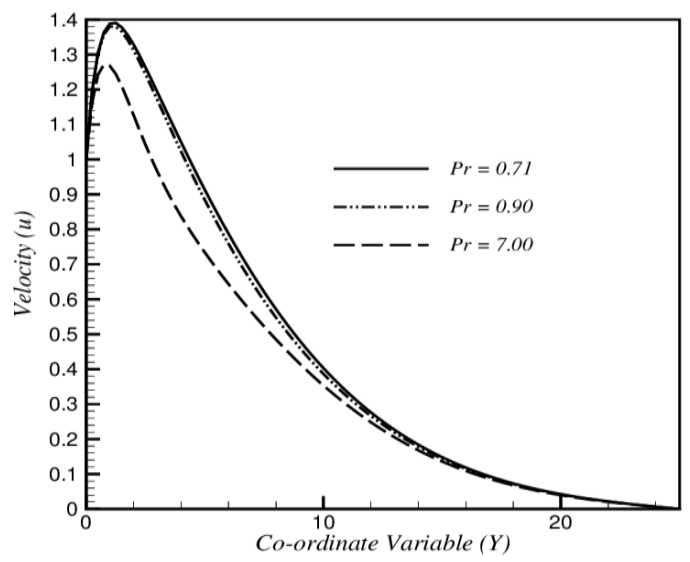

Fig. 2- Variation of velocity $u$ for different values of Prandtl number $\operatorname{Pr}$ at $\tau=10$ when $G r=2.0, G m=3.0$, $M=3.0, S=0.01, S_{o}=1.0$ and $S c=0.22$

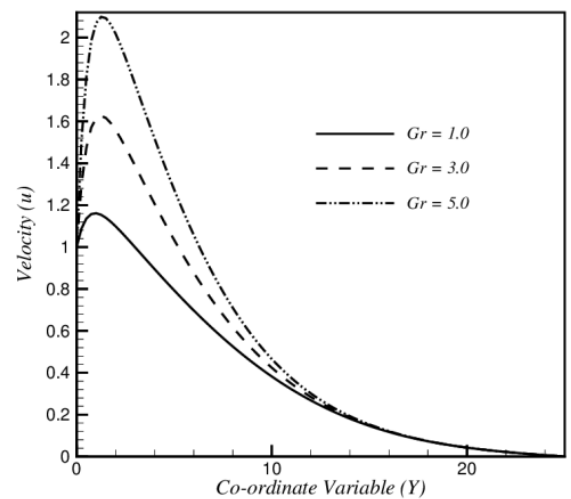

Fig. 4- Variation of velocity $u$ for different values of Grashof number $G r$ at $\tau=10$ when $G m=3.0, M=3.0, S_{o}$ $=1.0, S=0.01, P r=0.71$ and $S c=0.22$

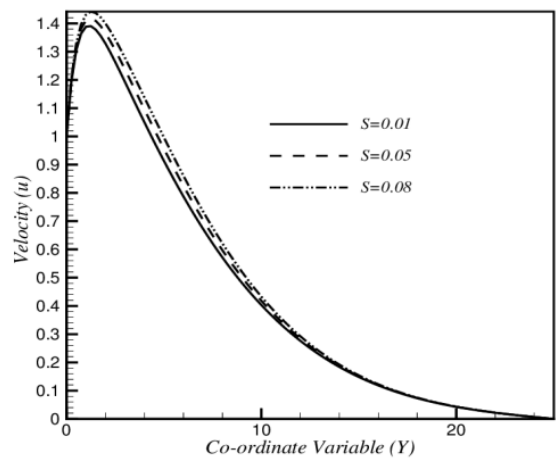

Fig. 6- Variation of velocity $u$ for different values of heat source parameter $S$ at $\tau=10$ when $G r=2.0$, $G m=3.0, M=3.0, S_{o}=1.0, S c=0.22$, and $P r=0.71$ 


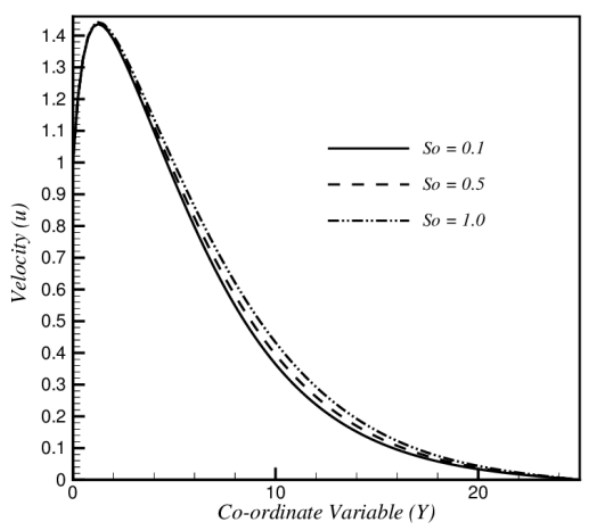

Fig. 7- Variation of velocity $u$ for different values of soret number $S o$ at $\tau=10$ when $G r=2.0, G m=3.0, M=3.0, S=0.08, S c=0.22$ and $\operatorname{Pr}=0.71$

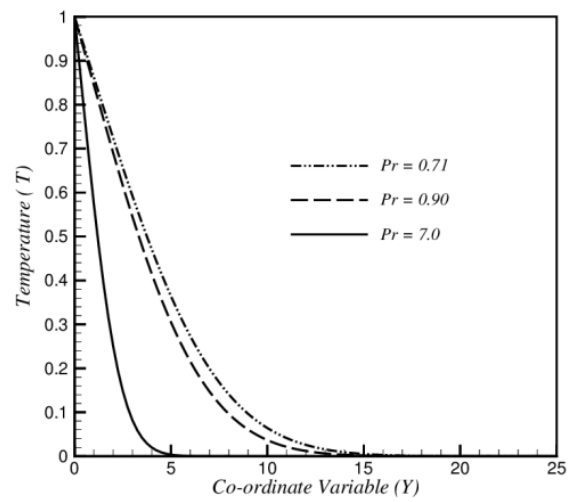

Fig. 9- Variation of temperature $T$ for different values of Prandtl number $\operatorname{Pr}$ at $\tau=10$ when $G r=$ 3.0, $G m=3.0, M=3.0, S=0.01, S_{o}=1.0$ and $S c$ $=0.22$

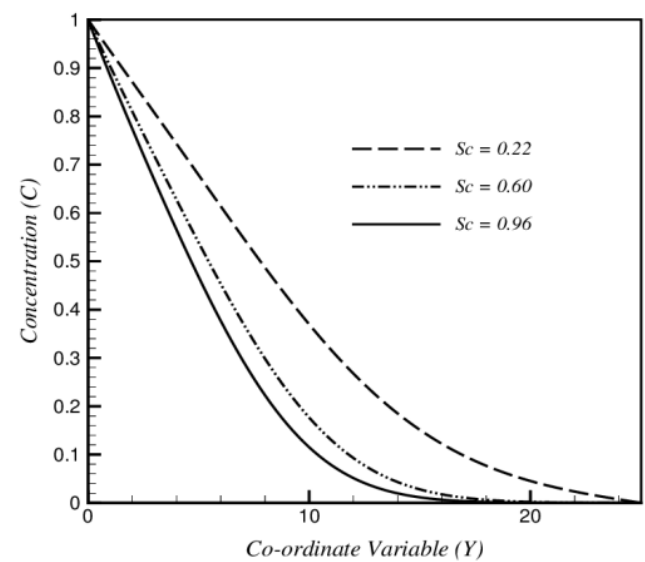

Fig. 11- Variation of concentration $C$ for different values of Schmidt number $S c$ at $\tau=10$ when $G r=3.0, G m=3.0, M=3.0, \quad P r=0.71$, $S_{0}=1.0$ and $S=0.01$

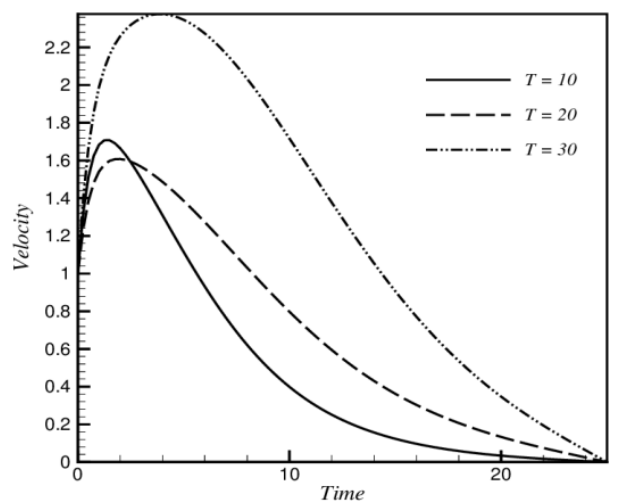

Fig. 8- Variation of velocity $u$ for different values of time $\tau$ when $G r=3.0, G m=3.0, M=3.0, S=0.08$, $S c=0.22$, So $=0.1$ and $P r=0.71$

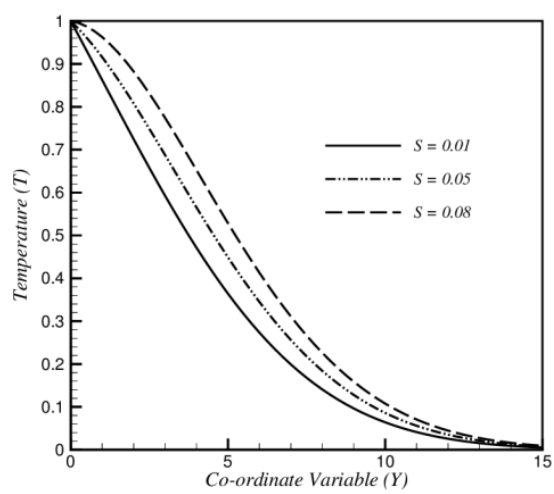

Fig. 10- Variation of temperature $T$ for different values of heat source parameter $S$ at $\tau=10$ when $G r=3.0, G m=3.0, M=3.0, \quad S_{0}=1.0, S c=0.22$ and $\operatorname{Pr}=0.71$

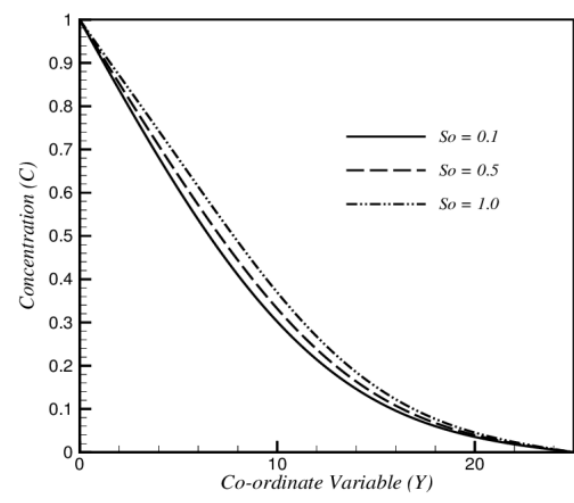

Fig. 12-Variation of concentration $C$ for different values of soret number $S o$ at $\tau=10$ when $G r=3.0$, $G m=3.0, M=3.0, P r=0.71, S c=1.0$ and $S=0.01$ 


\section{CONCLUSION}

From the study of the paper we conclued that the velocity are incrising with the incrising value of Grashof number $(\mathrm{Gr})$, Soret number $\left(S_{o}\right)$ and modified Grashof number $(\mathrm{Gm})$ on the other hand it is decrising with the incrising value of magnetic parameter $(M)$, Prandtl number $(P r)$, Schmidt number $(S c)$. We see that if the time is increasing then the velocity is incrising. The temperature increase with increase of heat source parameter $(S)$ and decrease with increase of Prandtl number $(P r)$. Also we see that the concentration is increase with the increase of Soret number $\left(S_{0}\right)$ and decrease with the increase of Schmidt number $(S c)$.

\section{REFERENCES}

[1] R.K Singh and A.K Singh, Int. J. of Appl. Math and Mech. 10.1007/s10483-012-1616-7

[2] R. C. Chaudhary and J. Arpita, Roman J. Phy., Volume 52, Nos. 5-7, P. 505-524, Bucharest, 2007

[3] B. P. Reddy and J. A. Rao, Int. J. of Applied Math and Mech. 7(8): 78-97, 2011

[4] A. K. Singh, A. K. Singh and N.P. Singh, Bulletin of the institute of mathematics academia since, Volume 33, number 3, September 2005

[5] B. Gebhart, Heat Transfer. New York: Mc Graw-Hill Book Co., 1971.

[6] B. Gebhart and L. Pera, Ind. J. Heat Mass Transfer, pp. 2025-2050, 14, 1971

[7] A. A. Raptis and V. M. Soundalgekar, ZAMM, pp. 127-130, 64, 1984

[8] B. K. Jha and A. K. Singh, Astrophysics. Space Science, pp. 251-255, 173, 1990

[9] M. D. Abdusattar, Ind. J. Pure Applied Math., pp. 259-266, 25, 1994

[10] M. Acharya, G. C. Das and L. P. Singh, Ind. J. Pure Applied Math., pp. 1- 18, 31,2000

[11] A. Bejan and K. R. Khair, Int. J. Heat Mass Transfer, pp. 909-918, 28, 1985

[12] M. S. Alam and M. M. Rahman, BRACK University J., Volume II, No.1, pp.111-115, 2005

[13] N. P. Shingh, A. K. Shingh and A. K. Shingh, The Arabian J. for Science and Engineering, Volume 32, Number 1A January 2007 